\title{
What Type of Process Underlies Options? A Simple Robust Test
}

\author{
PETER CARR \\ New York University \\ LIUREN WU \\ Fordham University
}

Follow this and additional works at: https://fordham.bepress.com/crif_working_papers

Part of the Finance and Financial Management Commons

\section{Recommended Citation}

CARR, PETER and WU, LIUREN, "What Type of Process Underlies Options? A Simple Robust Test" (2002). CRIF Working Paper series. 20.

https://fordham.bepress.com/crif_working_papers/20 


\title{
What Type of Process Underlies Options? A Simple Robust Test*
}

\author{
Peter CARR ${ }^{\dagger}$ \\ Courant Institute, New York University \\ LIUREN WU \\ Graduate School of Business, Fordham University
}

First draft: November 3, 2000

This version: June 19, 2002

${ }^{*}$ The authors thank George Chacko, Xiong Chen, Vladimir Dobric, Robert Engle, John Illuzi, Stewart Inglis, Keith Lewis, Dilip Madan, Andrew Mitchell, Nate Newlin, Ken Singleton, and participants of the 2002 American Financial Association meetings for helpful comments.

$\dagger 251$ Mercer Street, New York, NY 10012; tel: (212) 260-3765; pcarr@nyc.rr.com.

$\$ 113$ West 60th Street, New York, NY 10023; tel: (212) 636-6117; fax: (212) 765-5573; wuefordham.edu; www. bnet. fordham. edu/ l wu. 


\title{
What Type of Process Underlies Options? A Simple Robust Test
}

\begin{abstract}
We develop a simple robust test for the presence of continuous and discontinuous (jump) components in the price of an asset underlying an option. Our test examines the prices of at-the-money and out-of-the-money options as the option maturity approaches zero. We show that these prices converge to zero at speeds which depend upon whether the sample path of the underlying asset price process is purely continuous, purely discontinuous, or a mixture of both. By applying the test to S\&P 500 index options data, we conclude that the sample path behavior of this index contains both a continuous component and a jump component. In particular, we find that while the presence of the jump component varies strongly over time, the presence of the continuous component is constantly felt. We investigate the implications of the evidence for parametric model specifications.
\end{abstract}

JEL Classification Codes: G12, G13, C52.

KEY WORDS: Jumps; continuous martingale; option pricing; Lévy density; double tails; local time. 
In continuous time finance, the asset price process is almost always modeled as a semimartingale (Delbaen and Schachermayer (1994)). By definition, every semimartingale can be uniquely decomposed into two components: a predictable component and a martingale component. Intuitively speaking, this decomposition amounts to decomposing every increment of the process into a conditional forecast and an innovation. In general, the innovation (martingale) component can either be a purely continuous martingale (henceforth PC), a pure jump martingale (henceforth PJ), or a combination of both (henceforth $\mathrm{CJ}$ ). While the traditional modeling effort has mainly focused on purely continuous processes, growing attention has been given to pure jump processes and combinations of continuous and jump processes. Examples include, among others, Aït-Sahalia, Wang, and Yared (2001), Andersen, Benzoni, and Lund (2002), Bates (1991), Duffie, Pan, and Singleton (2000), and Merton (1976).

Despite the important implications for asset pricing and for risk management, it remains an open question as to which of the three types of martingales drives the price movement of an asset. Most recently, Aït-Sahalia (2002) refines our understanding of the answer to this question. By applying the concept of total positivity, Aït-Sahalia (2002) shows that the cross second derivative of the transition density of a one-factor diffusion process has to be positive at all states and at all sampling intervals. He constructs a diffusion criterion based on such a property and applies the test to the risk-neutral transition density of the S\&P 500 index implied from observed option prices. The test rejects the hypothesis that the index follows a one factor diffusion process. However, since the test is constructed under the onefactor Markovian setting, his diffusion criterion could also be violated if the index follows a continuous sample path, but is not Markovian in itself. For example, in the presence of stochastic volatility, the single factor Markovian property is violated and thus the criterion can be violated even if the asset price follows a continuous sample path. Indeed, the presence of stochastic volatility in asset returns is well documented. See, for example, Bakshi, Cao, and Chen (1997), Bates (1996, 2000), Ding and Granger (1996), and Pan (2002).

This article proposes a procedure for identifying the nature of the martingale component under a much more general setting. In particular, the price process can be non-Markovian and can be driven by multiple factors such as stochastic volatilities. Our test does not look directly at the transition density 
Table I

\section{Behavior of Short-Maturity Options}

\begin{tabular}{ccc}
\hline \hline Model Type & OTM Options & ATM Options \\
\hline PC & $O\left(e^{-1 / T}\right)$ & $O(\sqrt{T})$ \\
PJ & $O(T)$ & $O\left(T^{p}\right), p \in(0,1]$ \\
CJ & $O(T)$ & $O\left(T^{p}\right), p \in(0,1 / 2]$ \\
& & \\
\hline \hline
\end{tabular}

nor at the sample path of the asset price. Instead, the test examines the prices of at-the-money (ATM) and out-of-the-money (OTM) options on the asset as the option maturity approaches zero. While the prices of all ATM and OTM options converge to zero as time to maturity decreases, our theoretical work shows that the speed of convergence differs across the three model types (PC, PJ, and CJ) and the two moneyness modes (ATM and OTM). This speed of convergence is described conveniently using Landau's notation, so that $f=O(g)$ implies $\lim \sup (f / g)<\infty$. Table I summarizes our theoretical results regarding the speed with which an option's premium approaches zero as time to maturity $T$ vanishes.

The table indicates that OTM option prices converge to zero at an exponential rate, $O\left(e^{-1 / T}\right)$, in the case of a purely continuous sample path (PC), but are dominated by a linear convergence rate, $O(T)$, in the presence of jumps. The table also shows that ATM option prices approach zero at a particular speed, $O(\sqrt{T})$, in the case of a purely continuous sample path. In contrast, ATM option prices can approach zero at a range of speeds in the case of a pure jump process (PJ). If the jump process has sample paths with finite variation, e.g., a compound Poisson process with a possibly random jump size and potentially time-varying (but finite) jump intensity, the power $p$ in the table is one. However, if the jump process has sample paths of infinite variation, the order of convergence can be anything between zero and one. In the case of a combination of both continuous and discontinuous sample paths (CJ), the convergence rate is dominated by the component with the slowest convergence to zero. Thus, observations of the convergence rates of option prices to zero can potentially be used to distinguish the type of the martingale component of the underlying asset price process. 
The different decay speeds experienced by option premiums are most easily visualized by a graph. In this paper, we focus on a graph which plots the log of the ratio of option prices to maturity against log maturity. We christen such a graph as the term decay plot. The division of option prices by maturity is used to visually contrast order $O(T)$ behavior from sub $O(T)$ and super $O(T)$ behavior. For OTM options, the asymptotic behavior of the term decay plots (as maturity approaches zero) determines whether or not jumps are being priced into options. In particular, an asymptotic slope of 0 for the OTM term decay plot implies the existence of a jump component, while an asymptotic slope of positive infinity is consistent with a purely continuous sample path. For ATM options, an asymptotic slope of 0 would imply a pure jump process with finite variation. An asymptotic slope of -0.5 implies that either the sample path has a continuous component, or that the jump component exhibits infinite variation (or both). An asymptotic slope of any other value implies the existence of an infinite variation pure jump process, which can also be masking a continuous component if the asymptotic slope is below -0.5 , eg., -0.6 .

The theoretical results in Table I are based on the asymptotic properties of option prices as the option maturity approaches zero. To determine at what maturity range we can observe the asymptotic behaviors, we simulate several parametric models under each of the three process types. For all models simulated, we find that the theoretical asymptotic behavior is always experienced by options maturing within 20 days. In some cases, the asymptotic behavior is experienced over a much longer term. For example, for ATM options in models with a continuous martingale component, the asymptotic behavior is experienced by options maturing within a year. Options with maturities from one week to one year are readily available and liquid for many underlying assets such as stocks, stock indices, currencies, bonds, etc. Thus, the types of the sample paths of these assets can readily be tested using market prices of their respective options.

We apply the test to S\&P 500 index options by estimating the term decay plots at different moneyness levels for more than one year's worth of daily closing quotes. We find that the sample path of the index contains both a continuous and discontinuous martingale components. In particular, we find that while the presence of the jump component varies strongly over time, the presence of the continuous 
component is constantly felt. We investigate the implications of the evidence for parametric model specifications.

Our theoretical framework and our proposed test focus on the risk-neutral dynamics of an underlying asset. However, under no arbitrage, the type of process found under a risk-neutral measure is preserved under a measure change to the statistical measure. Hence, our findings have implications for both pricing and risk management. More specifically, our findings indicate that risk measurement and management should be conducted under the assumption that the real world process has both continuous and discontinuous martingale components, with the relative weight of the two components varying over time.

Our test presents interesting contrasts to the test proposed by Aït-Sahalia (2002). First, Aït-Sahalia (2002) tests whether the underlying asset follows a one-factor (Markovian) diffusion process or not; our test is designed to identify the presence of a jump component and/or a continuous component and is not confined to a one factor or Markovian set-up. Second, Aït-Sahalia (2002) looks at the transition density across all potential states at any fixed time horizon. In contrast, our test looks at the option price behavior across maturities at fixed moneyness (states). Hence, the two tests complement each other by focusing on different dimensions of the information set.

The remainder of this paper is organized as follows. The next section develops our theoretical results underlying Table I. Section II simulates popular model candidates under each of three model types and analyzes at which maturity options can be characterized by their asymptotic behaviors. Section III applies the test to S\&P 500 index options. Section IV concludes.

\section{Theory of Short Maturity Option Pricing}

\section{A. Assumptions and Notation}

We assume frictionless markets and no arbitrage. Then, under a risk-neutral measure $\mathbb{Q}$, the return on an asset can be modeled as a superposition of a predictable drift component and a martingale. The 
drift component is determined by no arbitrage. The martingale component can further be decomposed canonically into two orthogonal components: a purely continuous martingale and a purely discontinuous martingale (Jacod and Shiryaev (1987), page 84).

To fix notation, let $S_{t}$ denote the spot price of an asset at time $t \in[0, \mathcal{T}]$, where $\mathcal{T}$ is some arbitrarily distant horizon. No arbitrage implies that there exists a risk-neutral probability measure $\mathbb{Q}$ defined on a probability space $(\Omega, \mathcal{F}, \mathbb{Q})$ such that the spot price solves the following stochastic differential equation,

$$
d S_{t} / S_{t-}=(r-q) d t+\sigma_{t} d W_{t}+\int_{\mathbb{R}^{0}}\left(e^{x}-1\right)\left[\mu(d x, d t)-v_{t}(x) d x d t\right], \quad t \in[0, \mathcal{T}]
$$

starting at some fixed and known value $S_{0}>0$. In (1), $S_{t-}$ denotes the asset price at time $t$ just prior to a jump, $\mathbb{R}^{0}$ denotes the real line excluding zero, $r$ and $q$ are, respectively, the continuously compounded risk free rate and dividend yield, $W_{t}$ is a $\mathbb{Q}$ standard Brownian motion, and the random measure $\mu(d x, d t)$ counts the number of jumps of size $x$ in the asset price at time $t$. The process $\left\{\mathrm{v}_{t}(x), x \in \mathbb{R}^{0}, t \in[0, \mathcal{T}]\right\}$ compensates the jump process $J_{t} \equiv \int_{0}^{t} \int_{\mathbb{R}^{0}}\left(e^{x}-1\right) \mu(d x, d s)$, so that the last term in (1) is the increment of a $\mathbb{Q}$-pure jump martingale. ${ }^{1} v_{t}(x)$ is often referred to as the compensator or the local density of the jumps. Thus, equation (1) models the price change as the sum of a risk-neutral drift and two martingale components: a purely continuous martingale and a purely discontinuous (jump) martingale.

To avoid the notational complication of truncation functions, we assume that the jump process exhibits finite variation,

$$
\int_{\mathbb{R}^{0}}(|x| \wedge 1) v_{t}(x) d x<\infty, \quad t \in[0, \mathcal{T}]
$$

We later relax this assumption and discuss the case of infinite variation jump processes separately. By adding the time subscripts to $\sigma_{t}$ and $v_{t}(x)$, we allow both to be stochastic and predictable with respect to the filtration $\mathcal{F}_{t}$. To satisfy limited liability, we assume the two stochastic processes to be such that

\footnotetext{
${ }^{1}$ The process $v_{t}(x)$ must have the following properties (see Prokhorov and Shiryaev (1998)),

$$
v_{0}(x)=0, \quad v_{t}(0)=0, \quad \int_{\mathbb{R}^{0}}\left(|x|^{2} \wedge 1\right) v_{t}(x) d x<\infty, \quad t \in[0, \mathcal{T}]
$$
}


the asset price $S_{t}$ is always nonnegative and absorbing at the origin. We further assume that the explicit time dependence of $\sigma_{t}$ and $v_{t}$ has a leading term of order zero.

In principle, we can also allow the interest rate and dividend yield to be stochastic. Nevertheless, given their lesser role in option pricing, we confine both to be constant over time with little loss of generality. Then, the forward price of the asset at a fixed maturity date $T, F_{t}=S_{t} e^{(r-q)(T-t)}$, is a $\mathbb{Q}$-martingale with the following dynamics,

$$
d F_{t} / F_{t-}=\sigma_{t} d W_{t}+\int_{\mathbb{R}^{0}}\left(e^{x}-1\right)\left[\mu(d x, d t)-v_{t}(x) d x d t\right], \quad t \in[0, \mathcal{T}]
$$

where $F_{t-}$ denotes the pre-jump forward price at time $t$. It proves convenient to represent our theoretical results in terms of the forward price.

\section{B. Time Value Decomposition}

Consider a European call option with strike price $K$ and maturity $T$. Let time $t=0$ denote the valuation date and let $C_{0}(K, T)$ denote the time 0 price of the call option. Let $T V_{0}(K, T)$ denote the call option's time value, which is defined as

$$
T V_{0}(K, T) \equiv C_{0}(K, T)-e^{-r T}\left(F_{0}-K\right)^{+}
$$

where $F_{0}=S_{0} e^{(r-q) T}$ is the time- 0 forward price of the asset. The time value of a put option can be defined analogously. Obviously, the option price and time value coincide with each other for ATM and OTM options ( $K \geq F_{0}$ for call options and $K \leq F_{0}$ for put options) as they possess zero intrinsic value. Also, put-call parity implies that European put options and call options of the same strike and maturity have the same time value.

The main theoretical result of the paper comes from the following theorem, which decomposes the time value of a European option into two parts: the contribution from the continuous martingale component and that from the purely discontinuous martingale component. 
Theorem 1 For an asset price process characterized by (1), the time value of a European option on such an asset can be decomposed into two parts:

$$
T V_{0}(K, T)=e^{-r T} \int_{0}^{T}\left[\frac{1}{2} q\left(K, t_{-}\right) K^{2} \mathbb{E}_{0}\left[\sigma_{t}^{2} \mid F_{t-}=K\right]+\mathbb{E}_{0}\left[F_{t-} v_{t}^{o}(k)\right]\right] d t
$$

where $\mathbb{E}_{0}[\cdot]$ denotes expectation under the risk-neutral measure $\mathbb{Q}$ conditional on filtration $\mathcal{F}_{0}, q\left(K, t_{-}\right)$ denotes the $\mathbb{Q}$-probability density function of $F_{t_{-}}$evaluated at $F_{t_{-}}=K$, and $v_{t}^{o}(k)$ is the double tail of the local density defined as,

$$
v_{t}^{o}(k) \equiv \begin{cases}\int_{k}^{\infty}\left(e^{x}-e^{k}\right) \mathrm{v}_{t}(x) d x \quad \text { if } \quad k>0 \\ \frac{1}{2} \int_{\mathbb{R}^{0}}\left|e^{x}-1\right| \mathrm{v}_{t}(x) d x \quad \text { if } \quad k=0, \quad k \equiv \ln K / F_{t-} \\ \int_{-\infty}^{k}\left(e^{k}-e^{x}\right) \mathrm{v}_{t}(x) d x \quad \text { if } \quad k<0\end{cases}
$$

Note that the first part of the time value is related to the quadratic variation of the diffusion component, while the second part is a function of the compensator of the jump component. Also note that, when $k=0$, the double tail $v_{t}(0)$ is finite only for finite variation jumps. Nevertheless, $v_{t}(k)$ is finite for all jump types as long as $|k|$ is strictly bounded away from zero. The proof of the theorem follows from a decomposition of the terminal payoff function via the application of the Meyer-Tanaka formula (See, for example, Protter (1990), page 165), which extends Itô's lemma to functions that are not necessarily twice differentiable. We then take expectations to obtain the option value.

Proof. We start with a European call option on the asset with strike $K$ and maturity $T$. The terminal payoff of such an option is given by,

$$
\left(S_{T}-K\right)^{+}=\left(F_{T}-K\right)^{+}
$$

By the Meyer-Tanaka formula, the terminal payoff function in (5) can be decomposed as

$$
\left(F_{T}-K\right)^{+}=\left(F_{0}-K\right)^{+}+\int_{0}^{T} 1\left(F_{t-}>K\right) d F_{t}+\frac{1}{2} \int_{0}^{T} F_{t-}^{2} \sigma_{t}^{2} \delta\left(F_{t-}-K\right) d t
$$




$$
+\int_{0}^{T} \int_{\mathbb{R}^{0}}\left[1\left(F_{t-} \leq K\right)\left(F_{t-} e^{x}-K\right)^{+}+1\left(F_{t-}>K\right)\left(K-F_{t-} e^{x}\right)^{+}\right] \mu(d x, d t)
$$

where $\delta(\cdot)$ denotes a dirac density.

Taking expectations on both sides of (6) under measure $\mathbb{Q}$, we have

$$
\begin{aligned}
e^{r T} C_{0}(K, T)= & \left(F_{0}-K\right)^{+}+\frac{1}{2} \int_{0}^{T} \mathbb{E}_{0}\left[F_{t-}^{2} \sigma_{t}^{2} \delta\left(F_{t-}-K\right)\right] d t \\
& +\int_{0}^{T} \mathbb{E}_{0} \int_{\mathbb{R}^{0}}\left[1\left(F_{t-} \leq K\right)\left(F_{t-} e^{x}-K\right)^{+}+1\left(F_{t-}>K\right)\left(K-F_{t-} e^{x}\right)^{+}\right] v_{t}(x) d x d t
\end{aligned}
$$

Note that the expectation of the second term in (6), $\int_{0}^{T} 1\left(F_{t-}>K\right) d F_{t}$, is zero by the martingale property of $F_{t}$. Also note that we replace the random measure $\mu(d x, d t)$ in the jump term by its conditional expected value (compensator) $v_{t}(x) d x d t$ via the law of iterated expectations.

We further factor out $F_{t-}$ from the jump term in (7) and represent the term as a function of $k \equiv$ $\ln K / F_{t-}$, the moneyness of the option just prior to any jump at time $t$. Then, equation (3) follows after the following substitutions and rearrangements,

$$
\begin{aligned}
\mathbb{E}_{0}\left[F_{t-}^{2} \sigma_{t}^{2} \delta\left(F_{t-}-K\right)\right] & =q\left(K, t_{-}\right) K^{2} \mathbb{E}_{0}\left[\sigma_{t}^{2} \mid F_{t-}=K\right] \\
T V_{0}(K, T) & =C_{0}(K, T)-e^{-r T}\left(F_{0}-K\right) \\
v_{t}^{0}(k) & =\int_{\mathbb{R}^{0}}\left[1(k \geq 0)\left(e^{x}-e^{k}\right)^{+}+1(k<0)\left(e^{k}-e^{x}\right)^{+}\right] v_{t}(x) d x
\end{aligned}
$$

Finally, since a European put option has the same time value as a European call option with the same strike and maturity, equation (3) applies to both puts and calls.

The compensating process $v_{t}(j)$ can be interpreted as the probability per unit time of a jump of size $j$ at time $t$. More precisely,

$$
\lim _{\triangle T \downarrow 0} \frac{\mathbb{Q}\left\{\ln F_{t+\triangle T}-\ln F_{t-} \in d x\right\}}{\triangle T} \rightarrow v_{t}(x) d x
$$


where $\rightarrow$ denotes vague convergence on $\{|x|>\varepsilon\}$ for every fixed $\varepsilon>0$ (see Bertoin (1996), page 39). We label $v_{t}^{o}(k)$ as the double tail because it can also be written as the tail of the tail of the local density,

$$
\mathrm{v}_{t}^{o}(k)= \begin{cases}\int_{k}^{\infty} e^{i} \int_{i}^{\infty} \mathrm{v}_{t}(x) d x d i & \text { if } \quad k>0 \\ \frac{1}{2} \int_{0}^{\infty} e^{i} \int_{i}^{\infty} \mathrm{v}_{t}(x) d x d i+\frac{1}{2} \int_{-\infty}^{0} e^{i} \int_{-\infty}^{i} \mathrm{v}_{t}(x) d x d i & \text { if } \quad k=0 \\ \int_{-\infty}^{k} e^{i} \int_{-\infty}^{i} \mathrm{v}_{t}(x) d x d i & \text { if } \quad k<0\end{cases}
$$

As a corollary, the theorem also tells us the maturity derivative of ATM and OTM options.

Corollary 1 The maturity derivative of an at or out-of-the money option can be decomposed into three parts:

$$
\frac{\partial}{\partial T} T V_{0}(K, T)=e^{-r T} \frac{1}{2} q\left(K, T_{-}\right) K^{2} \mathbb{E}_{0}\left[\sigma_{T}^{2} \mid F_{T-}=K\right]+e^{-r T} \mathbb{E}_{0} F_{t-} v_{t}^{o}(k)-r T V_{0}(K, T) .
$$

This expression is often called the forward equation for option prices. Andersen and Andreasen (1999) arrive at a similar result under the assumption of the Poisson jump model of Merton (1976).

\section{Short Maturity Behavior}

This section considers the asymptotic behavior of ATM and OTM option prices as maturity approaches zero (i.e. $T \downarrow 0$ ). We first derive the asymptotic behavior based on Theorem 1, under the assumption that the jump component exhibits finite variation. We then consider the special case of infinite variation jumps.

\section{C.1. Continuous Martingale and Finite Variation Jumps}

The following proposition is a direct result of Theorem 1.

Proposition 1 As maturity approaches zero, option prices converge to zero at rates which depend upon both the moneyness and the type of the underlying price process. OTM option prices converge to zero 
at the rate of $O\left(e^{-1 / T}\right)$ in the case of a purely continuous process and at the rate of $O(T)$ in the presence of a jump component. ATM option prices converge to zero at the rate of $O(\sqrt{T})$ in the purely continuous case and at the rate of $O(T)$ in the case of pure finite variation jump. The convergence rate is dominated by the lower order of the two in the case of a mixture process.

Proof. As $T \downarrow 0$, equation (3) implies that the time value of an option can be approximated as

$$
T V_{0}(K, T) \sim T\left[\frac{1}{2} q\left(K, T_{-}\right) K^{2} \sigma_{0}^{2}+F_{0} v_{0}^{o}(k)\right]
$$

where $f \sim g$ implies $\lim (f / g)=1$. We drop the interest rate discounting term $e^{-r T}$ because it converges to one as $T \downarrow 0$. We also drop the expectation operations on $\sigma_{t}^{2}$ and $v_{t}^{o}$, given that both are predictable with respect to the filtration $\mathcal{F}_{t}$.

We first consider OTM options $\left(K \neq F_{0}\right)$. In the case of a purely continuous process $\left(v_{0}^{o}=0\right)$, the time value reduces to

$$
T V_{0}(K, T) \sim T \frac{1}{2} q(K, T) K^{2} \sigma_{0}^{2}
$$

Hence, the order of decay depends on the density function $q(K, T)$. But as $T \downarrow 0$, all diffusion processes behave like a standard Brownian motion (see Varadhan (1967)). The probability density function approaches the following Gaussian density,

$$
q(K, T) \sim \frac{1}{\sqrt{2 \pi T} F_{0} \sigma_{0}} \exp \left(-\frac{\left(F_{0}-K\right)^{2}}{2 F_{0}^{2} \sigma_{0}^{2} T}\right), \quad K \neq F_{0} .
$$

Therefore, asymptotically, the time value decays at an exponential rate in this purely continuous case,

$$
T V_{0}(K, T) \sim \frac{\sqrt{T} K^{2} \sigma_{0}}{2 \sqrt{2 \pi} F_{0}} \exp \left(-\frac{\left(F_{0}-K\right)^{2}}{2 F_{0}^{2} \sigma_{0}^{2} T}\right) \sim \sqrt{T} O\left(e^{-1 / T}\right)
$$

where $O(\cdot)$ denotes the order of the decay in terms of maturity. In the case of a pure jump process $\left(\sigma_{0}^{2}=0\right)$, the time value reduces to

$$
T V_{0}(K, T) \sim T F_{0} v_{0}^{o}(k) \sim O(T)
$$


In the mixture case, the decay rate is dominated by that of the jump component, $O(T)$.

For ATM options $\left(K=F_{0}\right)$, the time value formula in (10) still holds. But the probability density function $q(K, T)$ is reduced to ${ }^{2}$

$$
q(K, F) \sim \frac{1}{\sqrt{2 \pi T} F_{0} \sigma_{0}}, \quad K=F_{0} .
$$

The decay rate implied by a purely continuous process is therefore $O(\sqrt{T})$. In the case of a pure finite variation jump process, the asymptotic rate of $O(T)$ still holds.

\section{C.2. Infinite Variation Jumps}

Theorem 1 and hence Proposition 1 are derived under the assumption that the jump martingale component, if it is present, exhibits finite variation. In particular, the at-the-money double tail $v_{t}^{o}(0)$ in (4) is finite only under the assumption of finite variation,

$$
\int_{\mathbb{R}^{0}}(|x| \wedge 1) \mathrm{v}_{t}(x) d x<\infty, \quad t \in[0, \mathcal{T}]
$$

The following proposition considers the asymptotic behavior of option prices when the underlying asset price process follows a pure infinite variation jump process.

Proposition 2 Suppose that the underlying asset price process is driven by a pure jump martingale with infinite variation. Then, ATM option prices can converge to zero at a range of speeds, $O\left(T^{p}\right)$, where the order $p \in(0,1)$. OTM options converge to zero at the rate of $O(T)$, the same as the case with finite variation jumps.

Table I summarizes the results in Propositions 1 and 2.

Proof. For OTM options, i.e., for moneyness $|k|=\left|\ln \left(K / F_{0}\right)\right|$ strictly bounded away from zero, the double tail $v_{0}^{o}(k)$ in (4) is well-defined even if the jump process exhibits infinite variation. Thus, the

\footnotetext{
${ }^{2}$ Brenner and Subrahmanyam (1988) derive a similar result under the Black-Scholes setting.
} 
order $O(T)$ decay rate for finite variation jumps proved in Proposition 1 extends to infinite variation jumps.

For ATM options, the double tail $v_{t}^{o}(0)$ in (4) is not well-defined for jump processes with infinite variation. We re-define the ATM double tail $v_{t}^{o}(0)$ as

$$
v_{t}^{o}(0)=\frac{1}{2} \int_{\mathbb{R}^{0}}\left|e^{x}-1-x 1_{|x|<1}\right| v_{t}(x) d x
$$

where the truncation is needed to keep the double tail finite. But the truncated small jumps also induce a different order of time dependence. In particular,

$$
\lim _{T \downarrow 0} \int_{0}^{T} \mathbb{E}_{0} \int_{|x|<1}|x| v_{t}(x) d x d t=\lim _{T \downarrow 0} \int_{|x|<1}|x| \mathbb{Q}\left\{\Delta \ln F_{t \in(0, T]} \in d x\right\} \sim O\left(T^{p}\right), \quad p \in(0,1),
$$

where $\Delta \ln F_{t \in(0, T]}$ denotes the jumps in $\ln F$ during the period $t \in(0, T]$. The fact that $\int_{|x|<1}|x| v(x) d x=$ $\infty$ for infinite variation jumps implies the order $p$ is smaller than one. The requirement that time value increases with maturity demands that the order $p$ be positive. The $O\left(T^{p}\right)$ decay rate induced by the small jumps dominates the $O(T)$ decay rate induced by large jumps for ATM options.

The behavior of infinite variation jump martingales can be further illustrated via the classical example of an $\alpha$-stable Lévy motion. In particular, the finite moment log stable (LS) model of Carr and Wu (2002) uses the $\alpha$-stable Lévy motion with maximum negative skewness as the martingale component of the risk-neutral process for asset prices,

$$
d S_{t} / S_{t-}=(r-q) d t+\sigma d L_{t}^{\alpha,-1}, \quad t \in[0, \mathcal{T}], \alpha \in(1,2), \sigma>0
$$

where the increment $d L_{t}^{\alpha, \beta}$ has an $\alpha$-stable distribution with zero drift, dispersion of $d t^{1 / \alpha}$, and a skewness parameter $\beta$ : $L_{\alpha}\left(0, d t^{1 / \alpha}, \beta\right)$. Setting $\beta=-1$ in the LS model guarantees the existence of a martingale measure and the finiteness of call option values. 
A key feature of the $\alpha$-stable Lévy motion is its self-similar property: $L_{t}^{\alpha, \beta}$ and $t^{1 / \alpha} L_{1}^{\alpha, \beta}$ possess the same distribution. Based on this property, we prove the following asymptotic behavior for ATM options under the LS model. ${ }^{3}$

Proposition 3 Under the LS model in (14), ATM option prices converge to zero at the rate of $O\left(T^{1 / \alpha}\right)$.

Proof. Based on (14), the forward price $F_{T}$ is an exponential martingale,

$$
F_{T}=F_{0} e^{\mu T+\sigma L_{T}^{\alpha,-1}}=F_{0} e^{\mu T+\sigma T^{1 / \alpha} L_{1}^{\alpha,-1}}
$$

where $\mu=\sigma^{\alpha} \sec \frac{\pi \alpha}{2}$ is a convexity adjustment term, which is finite only when the $\alpha$-stable motion exhibits maximum negative skewness. The second equality is obtained by the self-similar property of the $\alpha$-stable Lévy motion.

Now consider the value of an ATM call option $\left(K=F_{0}\right)$,

$$
\begin{aligned}
T V_{0}\left(F_{0}, T\right) & =\mathbb{E}_{0}\left[\left(F_{T}-F_{0}\right)^{+}\right]=F_{0} \mathbb{E}_{0}\left[\left(e^{\mu T+\sigma T^{1 / \alpha} L_{1}^{\alpha,-1}}-1\right)^{+}\right] \\
& =F_{0} \int_{-\frac{\mu}{\sigma} T^{1-1 / \alpha}}^{\infty}\left(e^{\mu T+\sigma T^{1 / \alpha} x}-1\right) q(x) d x
\end{aligned}
$$

where $q(x)$ denotes the probability density function of a standardized $\alpha$-stable random variable with zero mean and unit dispersion.

By Taylor expansion, one can show that

$$
\lim _{T \downarrow 0} \frac{\exp \left(\mu T+\sigma T^{1 / \alpha} x\right)-1}{T^{1 / \alpha}} \rightarrow \sigma x .
$$

Hence,

$$
\lim _{T \downarrow 0} \frac{T V_{0}\left(F_{0}, T\right)}{T^{1 / \alpha}} \rightarrow F_{0} \sigma \int_{0}^{\infty} x q(x) d x<\infty .
$$

Therefore, ATM option prices converge to zero at the rate of $O\left(T^{1 / \alpha}\right)$.

\footnotetext{
${ }^{3}$ We thank Xiong Chen for much of this proposition.
} 
An $\alpha$-stable Lévy motion is fully characterized by its Lévy measure,

$$
v(x)=c_{ \pm} x^{-\alpha-1}
$$

where $c_{+}$and $c_{-}$apply to the cases of $x>0$ and $x<0$ respectively. The maximum negative skewness in the LS model is achieved by setting $c_{+}=0$ and hence by only allowing negative jumps. The admissible domain of the tail index $\alpha$ for an $\alpha$-stable motion is $\alpha \in(0,2]$. The LS model restricts that $\alpha>1$ so that the return has the support of the whole real line. An $\alpha$-stable Lévy motion exhibits infinite variation when $\alpha>1$ because the following integral is not finite,

$$
\int_{\mathbb{R}^{0}}(|x| \wedge 1) x^{-\alpha-1} d x=\infty
$$

Nevertheless, to maintain finite quadratic variation, i.e.,

$$
\int_{\mathbb{R}^{0}}\left(|x|^{2} \wedge 1\right) x^{-\alpha-1} d x<\infty
$$

the tail index $\alpha$ cannot be greater than two. Therefore, the LS model can generate a range of convergence speeds for ATM options, $O\left(T^{p}\right)$, for $p \in(1 / 2,1)$. As $\alpha$ approaches two, the $\alpha$-stable Lévy motion degenerates into a (continuous) Brownian motion and the asymptotic decay rate approaches $O(\sqrt{T})$.

We thus obtain very different behaviors for the short-term values of options as we vary the type of the underlying asset price process. For OTM options, as maturity approaches zero, the decay rate of the jump component, $O(T)$, dominates the decay rate of the continuous martingale component, $O\left(e^{-1 / T}\right)$, since for small $T, e^{-1 / T}<<T$. Thus, should the decay rate of OTM option values be $O(T)$, we can conclude that there exists a jump component in the underlying asset price dynamics. We can then determine the existence of an infinite variation component (a continuous process or infinite variation jump) from the short maturity behavior of ATM options. If the observed decay rate for ATM options is also $O(T)$, we can conclude that the underlying process is a pure jump process with finite variation. On 
the other hand, if the observed decay rate is of order $O(\sqrt{T})$, there may exist a continuous martingale component and/or a jump component with infinite variation.

Now, suppose that the actual behavior of the underlying has a jump component, and that marketmakers price options by inserting an implied volatility into the pure diffusion Black-Scholes formula. The assumption that the actual price process has jump components implies that the OTM option values decay as $O(T)$. From (11), we see that for OTM option values to decay at this rate, the implied volatility must approach infinity at rate $\sqrt{T} O\left(e^{1 / T}\right)$ as the maturity approaches zero. The fact that market-makers often abandon the use of implied volatility for OTM options at short maturities suggests that jumps are priced into options at short maturities. Our empirical work in Section III confirms this conjecture.

This last point also brings us back to one of our assumptions: although we allow $a_{t}$ and $v_{t}$ to be stochastic, we do assume that the leading term in the time expansion is of order zero. As a counter example, suppose that we modify the Black-Scholes model by allowing the volatility to vary over time at the previously conjectured rate $\sqrt{T} O\left(e^{1 / T}\right)$. Then, the OTM option values would decay as $O(T)$ even though the underlying price process is a purely continuous process. However, the explosive nature of models like this excludes themselves from our consideration.

\section{Simulation of Popular Models}

The different asymptotic behaviors for ATM and OTM options under different models can be best captured by a graph of $\ln (P / T)$ versus $\ln (T)$, where $P$ denotes the prices of ATM or OTM options and $T$ denotes maturity. We christen this graph as a term decay plot. Proposition 1 implies that as time to maturity approaches zero, the term decay plot for ATM options exhibits either a flat line in case of a finite variation pure jump model or a downward sloping straight line in the presence of a continuous martingale component or an infinite variation jump component. On the other hand, a similar plot for OTM options exhibits either a flat line in the presence of jumps or an upward sloping concave curve in the case of a purely continuous process. 
In this section, we simulate the behavior of several popular model candidates under each of the model types. We focus on when the asymptotic behavior of options will become transparent from the term decay plot. Since we intend to apply the test to the S\&P 500 index options market, the parameters of each simulated model are chosen to approximate the behavior of S\&P 500 index options. In all of the simulation analysis, we set the interest rate and dividend yield constant at the empirically determined averages of $r=5.96 \%$ and $q=1.31 \%{ }^{4}$ We focus on the behavior of OTM and ATM put values because in practice OTM put options are more liquid than OTM calls in the S\&P 500 index options market. For each model, we compute put option prices at four moneyness levels: $k=\ln (K / F)=0$, $-3.07 \%,-6.14 \%$, and $-9.20 \%$, and at a range of maturities: $\ln T=[-4,0]$ with an equal interval of 0.2 . This maturity range corresponds to option maturities from five business days to one year. We analyze the term decay plots over this maturity range at each of the four moneyness levels. In particular, to assess the slope and curvature of these term decay plots, ${ }^{5}$ we perform a second order polynomial fit to the plots,

$$
\ln (P / T)=a(\ln T)^{2}+b(\ln T)+c
$$

Then, the slope of the plot at a certain maturity $T$ is given by $2 a \ln (T)+b$ and the curvature is given by $2 a$. Table II reports the slope and curvature estimates of the term decay plots for all simulated models. ${ }^{6}$ The slopes are measured at the short end of the maturity: $\ln T=-4$. The second order polynomial fits the simulated term decay plots well, with R-squares for all simulated plots greater than 0.97.

\footnotetext{
${ }^{4}$ While choosing an interest rate and dividend yield close to data average mimics better the behavior of the S\&P 500 index options, our experimentation shows that setting both to zero generates almost the same qualitative shape for the term decay plots.

${ }^{5}$ We thank an anonymous referee for pointing out that the curvature of the term decay plot also contains valuable information.

${ }^{6}$ To save space, we only report the estimates on term decay plots at moneyness $k=0$ and $k=-9.20 \%$.
} 


\section{A. Purely Continuous Processes (PC)}

We consider two candidates for purely continuous processes: one is the benchmark model of Black and Scholes (1973); the other is its stochastic volatility extension by Heston (1993). The Black-Scholes model leads to a geometric Brownian motion for the stock price under the risk-neutral measure $\mathbb{Q}$,

$$
d S_{t}=(r-q) S_{t} d t+\sigma S_{t} d W_{t}
$$

with constant instantaneous volatility $\sigma$. Heston (1993) allows the volatility to be stochastic and assumes that the instantaneous variance rate $v=\sigma^{2}$ follows a square-root process under measure $\mathbb{Q}$,

$$
d v_{t}=\kappa\left(\theta-v_{t}\right) d t+\sqrt{\beta v_{t}} d Z_{t}
$$

where $Z_{t}$ is another standard Brownian motion with $\mathbb{E}\left[d W_{t} d Z_{t}\right]=\rho d t$. The option pricing formula for the Black-Scholes model is well-known. Option prices under the stochastic volatility model of Heston (1993) can be efficiently computed via the FFT method of Carr and Madan (1999), given the characteristic function of the $\log$ price relative $s_{T}=\ln \left(S_{T} / S_{0}\right)$,

$$
\phi(u) \equiv \mathbb{E}_{0}\left[e^{i u s_{T}}\right]=\exp \left[i u(r-q) T-b(T) v_{0}-c(T)\right]
$$

where $v_{0}$ is the current level of the instantaneous variance rate and the coefficients $\{b(T), c(T)\}$ are the following functions of maturity $T$,

$$
\begin{aligned}
b(T) & =\frac{2 \delta\left(1-e^{-\eta T}\right)}{2 \eta-\left(\eta-\kappa^{*}\right)\left(1-e^{-\eta T}\right)} \\
c(T) & =\frac{\kappa \theta}{\beta}\left[2 \ln \left(1-\frac{\eta-\kappa^{*}}{2 \eta}\left(1-e^{-\eta T}\right)\right)+\left(\eta-\kappa^{*}\right) T\right]
\end{aligned}
$$

with

$$
\eta=\sqrt{\left(\kappa^{*}\right)^{2}+2 \beta \delta}, \quad \kappa^{*}=\kappa-i u \sqrt{\beta} \rho, \quad \delta=\left(i u+u^{2}\right) / 2 .
$$



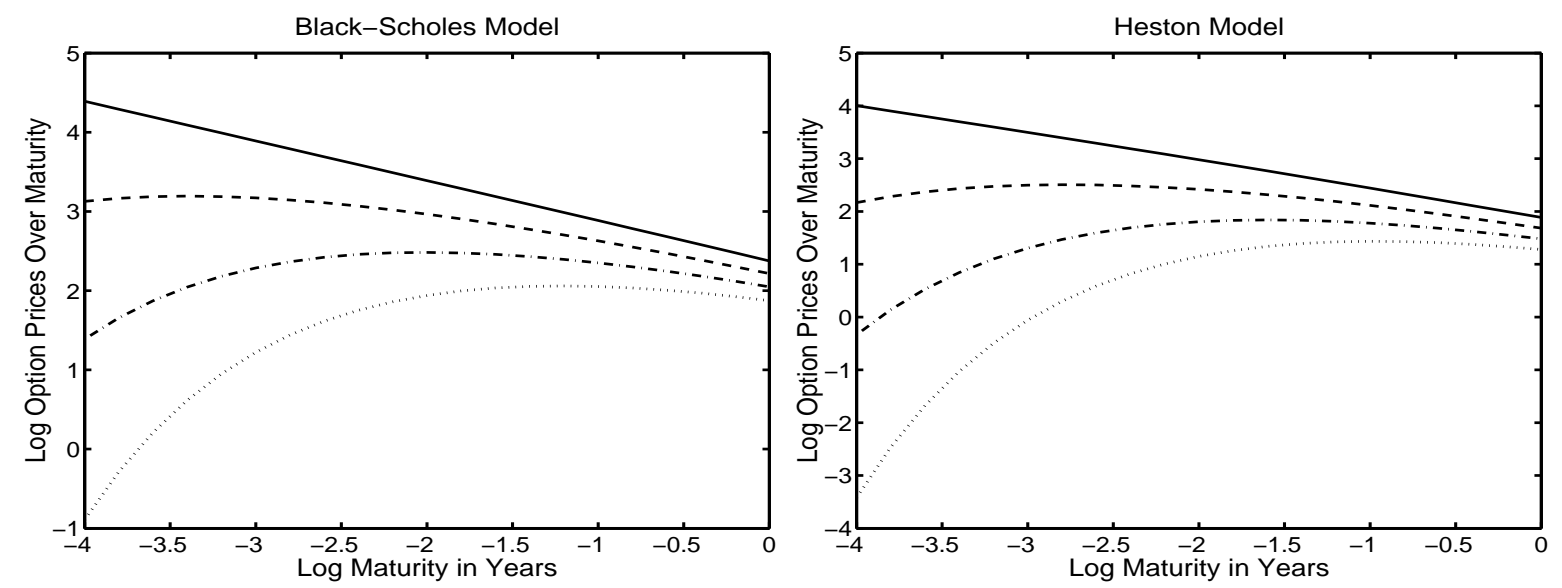

Figure 1. The Term Decay Plots Under Purely Continuous Processes

Lines are log put option prices over maturity plotted against log maturity. Option prices are computed from, in the left panel, the Black-Scholes model with $\sigma=27.4 \%$ and, in the right panel, the Heston (1993) model with $\theta=0.0348, \kappa=1.15, \beta=0.1521, \rho=-0.64, v_{0}=\theta$. We further assume spot price $S=100$, interest rate $r=5.96 \%$, and dividend yield $q=1.31 \%$. In each panel, the moneyness $k=\ln (K / F)$ is, from top to bottom, 0 (solid line), $-3.07 \%$ (dashed line), $-6.14 \%$ (dash-dotted line), and $-9.20 \%$ (dotted line).

Figure 1 depicts the term decay plots under the two purely continuous (PC) models. The volatility $\sigma$, in the Black-Scholes model is set to $27.4 \%$, a number close to the average of the implied volatility quotes on S\&P 500 index options in our sample period. The parameters for the Heston (1993) model are adopted from the estimates in Bakshi, Cao, and Chen (1997), who also calibrate the model to S\&P 500 index options. The term decay plots of the two PC models exhibit very similar behaviors. In particular, the plots for ATM options look like straight lines for both models as the term varies from five days to one year. Panel A of Table II reports the slope and curvature estimates of these term decay plots. The plots for ATM options show very little curvature $(-0.003$ and -0.018$)$ for the two PC models, and their short-maturity slope estimates $(-0.497$ and -0.493$)$ are close to the asymptotic theoretical value of $-1 / 2$. Thus, for purely continuous processes, regardless of whether stochastic volatility is present or not, the term decay plot for ATM options converges to its asymptotic behavior of a straight line at relatively long and hence readily observable maturities.

On the other hand, the term decay plots for OTM options are all upward sloping and concave, as expected from the asymptotic decay rate of $O\left(e^{-1 / T}\right)$ for continuous models. This behavior, particularly 
the upward sloping curve at short maturities, is more obvious for options deeper out of the money. The estimates in Table II confirm this observation. At moneyness $\ln (K / F)=-9.2 \%$, the term decay plots for both PC model exhibit strong concavity, with curvature estimates at -0.713 and -1.092 . Furthermore, the short-maturity slope estimates are large and positive, 1.988 for the Black-Scholes model and 3.158 for the Heston (1993) model.

\section{B. Pure Jump Processes (PJ)}

Under pure jump processes, OTM options converge to zero at the rate of $O(T)$ as time to maturity approaches zero. Hence, the term decay plot should converge to a flat line at short maturities. In contrast, the decay rate of ATM options depends upon whether the sample path of the jump process exhibits finite or infinite variation. In this subsection, we simulate two pure jump models, one with finite variation and the other with infinite variation. For the finite variation jump type, we simulate the most popular jump model, the compound Poisson jump model of Merton (1976) (MJ). While we recognize that $\mathrm{MJ}$ is usually implemented with a diffusion component, we investigate here the behavior of the pure jump version of his model. Under MJ, the arrival rate of jumps is controlled by a Poisson distribution with a constant and finite intensity $\lambda$. Conditional on one jump occurring, we assume that the size of the jump in the $\log$ price is drawn from a normal distribution with mean $\mu_{j}$ and variance $\sigma_{j}^{2}$. For the infinite variation jump type, we simulate the finite moment log stable (LS) model of Carr and $\mathrm{Wu}(2002)$ as described in (14). The driver of the process is an $\alpha$-stable Lévy motion with maximum negative skewness. The characteristic functions of the log returns under the two pure jump models are,

$$
\begin{aligned}
\phi_{M J}(u) & =\exp \left[i u\left(r-q-\lambda\left(e^{\mu_{j}+\frac{1}{2} \sigma_{j}^{2}}-1\right)\right) T+\lambda\left(e^{i u \mu_{j}-\frac{1}{2} u^{2} \sigma_{j}^{2}}-1\right) T\right] \\
\phi_{L S}(u) & =\exp \left[i u\left(r-q+\sigma^{2} \sec \frac{\pi \alpha}{2}\right) T-T(i u \sigma)^{\alpha} \sec \frac{\pi \alpha}{2}\right] .
\end{aligned}
$$

Figure 2 depicts the term decay plots implied by the two pure jump models at four different moneyness levels. Parameters are chosen to fit the general features of the S\&P 500 index options. ${ }^{7}$ The

\footnotetext{
${ }^{7}$ The parameters are adopted (but rounded off) from estimates in Carr and Wu (2002).
} 

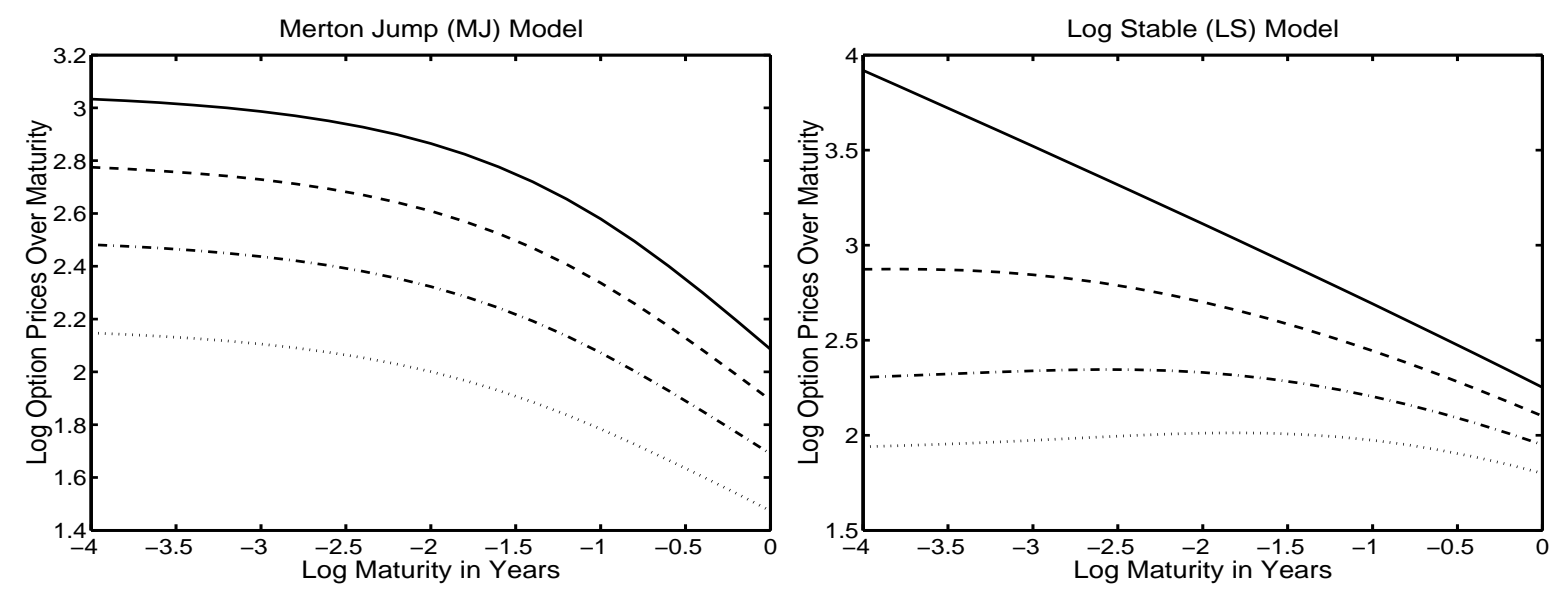

Figure 2. The Term Decay Plots Under Purely Discontinuous Processes

Lines are log put option prices over maturity plotted against log maturity. Option prices are implied by the MJ model in the left panel and the LS model in the right panel. The model parameters are set to: $\lambda=2, \mu_{j}=-0.10, \sigma_{j}=0.13$ for MJ, and $\sigma=0.15, \alpha=1.5$ for LS. We further assume spot price $S=100$, interest rate $r=5.96 \%$, and dividend yield $q=1.31 \%$. In each panel, the moneyness $k=\ln (K / F)$ is, from top to bottom, 0 (solid line), $-3.07 \%$ (dashed line), $-6.14 \%$ (dash-dotted line), and $-9.20 \%$ (dotted line).

term decay plots for OTM options are similar under both jump types and converge to their asymptotic behavior of a flat line as maturity falls within a month. As shown under panel B of Table II, for OTM options with moneyness $\ln (K / F)=-9.2 \%$, the short-maturity slope estimates for the term decay plots under both models are close to the asymptotic value of zero: 0.027 for MJ, and 0.085 for LS . The two plots also exhibit little curvature: -0.096 for MJ and -0.068 for LS.

In contrast, the term decay plots for ATM options exhibit distinct behaviors under the two types of pure jump processes. Under the finite variation MJ model, the ATM term decay plot flattens out as maturity falls within a month, similar to that for OTM options. The plot exhibits small concavity, with a curvature estimate of -0.155 , and the short-maturity slope estimate is 0.086 , very close to zero. On the other hand, under the infinite variation LS model, the ATM term decay plot cannot be visually distinguished from a straight line, similar to the behavior of a purely continuous process. The curvature estimate is very close to zero at -0.017 , and the short-maturity slope estimate is -0.357 , much closer to the asymptotic rate of a continuous component $(-0.5)$, than to the asymptotic rate of finite variation jumps (0). According to Proposition 3, under the LS model, the theoretical asymptotic decay rate for 

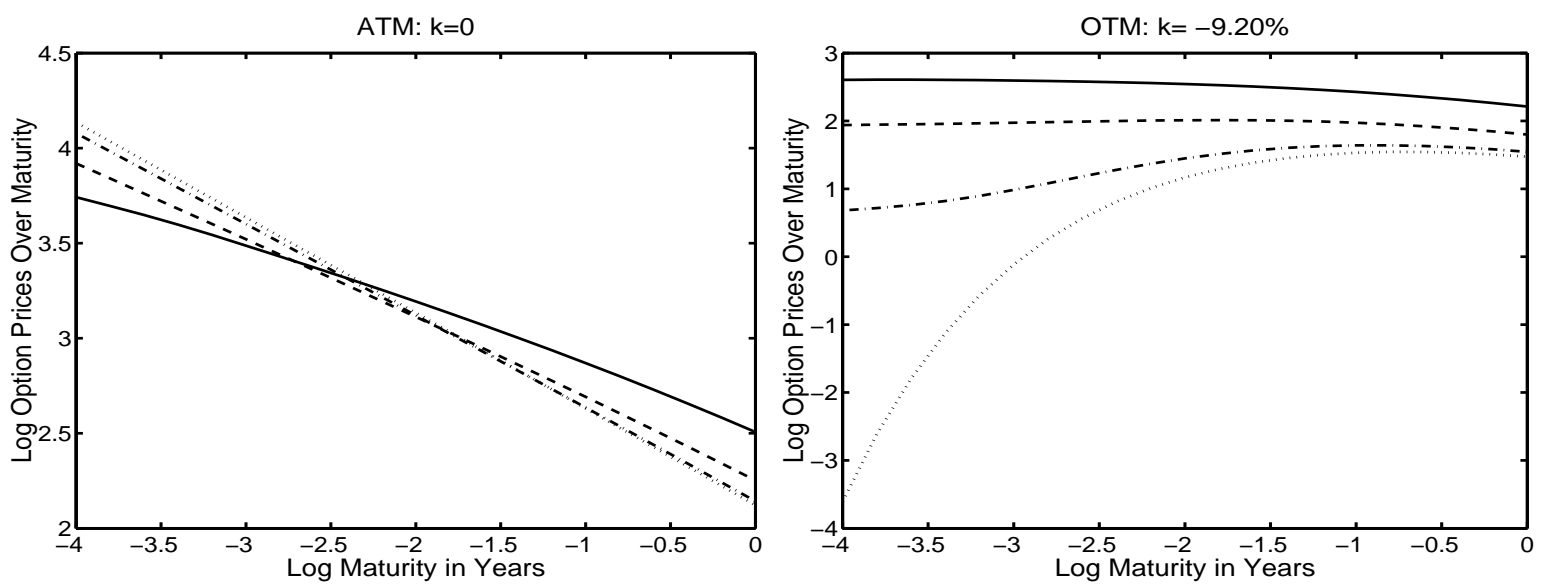

Figure 3. The Behavior of Option Prices Under the LS Model

Lines are log put option prices over maturity plotted against log maturity under the LS model of Carr and $\mathrm{Wu}$ (2002) with: $S=100, r=5.96 \%, q=1.31 \%, \sigma=15 \%$ and $\alpha$ equals, respectively, 1.2 (solid line), 1.5 (dashed line), 1.9 (dash-dotted line), and 2 (dotted line). The moneyness $k=\ln (K / F)$ is 0 (ATM) in the left panel and $-9.20 \%$ in the right panel.

the ATM term decay plot is $1 / \alpha-1$. Given that we set $\alpha=1.5$, the theoretical asymptotic rate is -0.333 .

While one can easily distinguish the ATM option behavior implied by a purely continuous process from a pure jump process with finite variation, the differences are not as easily discerned if the jump process also exhibits infinite variation. Furthermore, the infinite variation pure jump LS model degenerates into a pure diffusion model (the Black-Scholes model) as the tail index $\alpha$ approaches 2. Figure 3 further illustrates how, under the LS model, the behaviors of ATM and OTM option prices change at different values for the tail index $\alpha$. As shown in the left panel, all the plots for the ATM options look like straight lines: the curvature estimates are all close to zero. Furthermore, the short-maturity slope estimates, as reported in panel $\mathrm{C}$ of Table II, become closer to the asymptotic value of -0.5 of a continuous martingale as the tail index approaches two: The estimates are $-0.241,-0.357,-0.474$, and -0.498 for $\alpha=1.2,1.5,1.9,2$, respectively. ${ }^{8}$

\footnotetext{
${ }^{8}$ The corresponding theoretical asymptotic values are, respectively, $-0.167,-0.333,-0.474$, and -0.5 .
} 
For OTM options $(k=-9.20 \%$, right panel in Figure 3$)$, the distinction between a continuous ( $\alpha=2$, dotted line) and a jump $(\alpha<2)$ process looks more obvious. The short-maturity slope estimates are small at $0.044,0.085$, and 0.605 for $\alpha=1.2,1.5,1.9$, but increase to 3.255 as $\alpha=2$. Similarly, the curvature estimates of the plots are also small $(-0.068,-0.068$, and -0.170$)$ when $\alpha<2$ but are much larger $(-1.098)$ when $\alpha=2$. When $\alpha$ increases from 1.9 to 2 , the behaviors of the OTM options change dramatically.

\section{Combined Continuous Jump Processes (CJ)}

To each of the two pure jump models (MJ and LS) with the parameters in Figure 2, we add a continuous (diffusion) component with a constant instantaneous volatility of $14 \%$, which is about half of the average of the implied volatility quotes. The behavior of the combined models (MJD and LSD) are illustrated in Figure 4. The dominance of the diffusion component on the short maturity behavior of the ATM options is obvious, especially for models with finite variation jumps. For both models, the term decay plots for ATM options look more or less like straight lines. The curvature estimates of the ATM term decay plots for both models are close to zero: -0.019 for MJD and -0.005 for LSD (Panel D, Table II). Nevertheless, the short-maturity slope estimates for the plots are both smaller in absolute values than the asymptotic slope of the diffusion component $(-0.5)$. They are -0.347 for the MJD model and -0.452 for the LSD model. Hence, although the diffusion component is more dominating in the behavior of ATM options, the role of the jump component is still visible.

As the short maturity behavior of the OTM options are dominated by the jump component, the term decay plots for OTM options are very similar to those observed in Figure 2. The slope and curvature estimates of the plots for the MJ model and the MJD model are very close. Nevertheless, the diffusion component seems to have a visible impact on the OTM term decay plots under the LSD model. Under moneyness $\ln (K / F)=-9.2 \%$, incorporating a diffusion component makes the short maturity slope estimate slightly more positive from 0.085 for LS to 0.369 for LSD, and makes the curvature estimate slightly more neative from -0.068 for LS to -0.163 for LSD. Furthermore, simulation exercises (not 

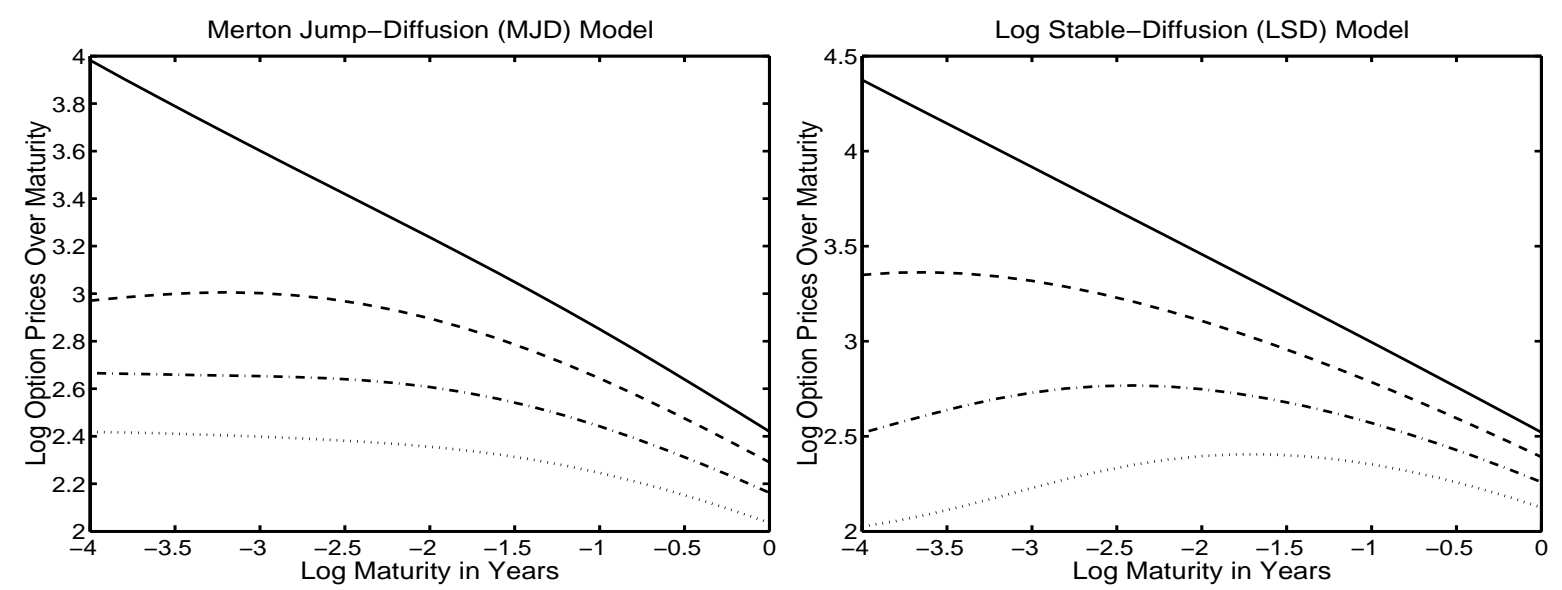

Figure 4. Term Decay Plots Under Mixture Models

Lines are log put option prices over maturity plotted against log maturity. Option prices are implied by a mixture of a diffusion component with a constant instantaneous volatility of $14 \%$ and a jump component. The jump component is MJ in the left panel and LS in the right panel with parameters the same as in Figure 2. In each panel, the moneyness $k=\ln (K / F)$ is, from top to bottom,0 (solid line), $-3.07 \%$ (dashed line), $-6.14 \%$ (dash-dotted line), and $-9.20 \%$ (dotted line).

reported) also indicates that the exact shape of the term decay plots are also affected by the relative magnitude of the two components.

In summary, as time to maturity approaches zero for OTM options, their price behavior is dominated by the presence of a jump component. This asymptotic dominance can be visually identified from options with maturities of 20 days or less. Thus, we can readily identify the presence of jumps in the underlying asset price movement from the short maturity behavior of OTM option prices. In addition, the short maturity behavior of the ATM options provides further information on the existence of an infinite variation component (from either a continuous or discontinuous process). The asymptotic dominance of this component on the behavior of ATM options becomes apparent as the option maturity falls within one year. 


\section{The Term Decay Plots for S\&P 500 Index Options}

We now turn to analyzing the behavior of the term decay plots for S\&P 500 index options, from which we infer the type of process the index follows.

\section{A. Data and Estimation}

The data on S\&P 500 index options are obtained from an options market maker. The data set contains the market maker's daily closing bid and ask price and implied volatility quotes on out-of-the-money options on S\&P 500 index spots across all strikes, $K$, and maturities, $T$, from April 6th, 1999 to May 31st, 2000 (290 business days). The data set also includes the matching daily closing futures prices $F$, spot index levels $S$, and interest rates $r$ corresponding to each option quote. We apply the following filters to the data: (1) the time to maturity is greater than five business days; (2) the bid option price is strictly positive; (3) the ask price is no less than the bid price. After applying these filters, we also plot the mid implied volatility for each day and maturity against strike prices to visually check for obvious outliers. After removing these outliers, we have 62,950 option quotes left over a period of 290 business days. From this data, we construct term decay plots for S\&P 500 index options and test the type of process the index follows.

We filter out very short maturities contracts and zero-bid contracts to minimize the impact of microstructure effects on the quotes. Our visual plot-by-plot inspection further removes the potential impact of data outliers. A potential concern of short maturity options is the synchronicity between option quotes and the underlying index levels. This issue is partially removed in our data set as the market maker also provides a matching implied volatility quote for each option, which, in general, does not vary as much with the underlying spot level as the option price does. In converting implied volatilities to option prices, we further normalize the option price as percentages of the underlying futures price. This normalization makes the term decay plots less sensitive to the underlying price movement, thus facilitating intertemporal comparison. 
For ease of comparison with the simulated models, we construct the term decay plots for S\&P 500 index options under the same moneyness levels as those in the simulation plots (Figures 1 to 4 ): $k=\ln (K / F)=0 \%,-3.07 \%,-6.14 \%$, and $-9.20 \%$. However, the observed options do not always correspond to these moneyness. In particular, since the forward price $F$ is varying every day while the strike prices $K$ are fixed, the moneyness $k=\ln (K / F)$ are varying over time. Thus, we need to interpolate across strike prices to obtain option prices at these fixed moneyness levels. For the interpolation to work with sufficient precision, we require that at each day and maturity, we have at least five option quotes. We do not extrapolate. Visual inspection indicates that at each date and maturity, the quotes are so close to each other along the moneyness line that interpolation can be done with little error, irrespective of the interpolation method. For the reported results, we use spline interpolation on put option prices across moneyness $k$ at each maturity and date. We have also experimented with several different interpolation schemes and on different spaces. The results are almost identical.

Given the interpolated option prices at each fixed moneyness level, we construct a smoothed term decay plot at each of the four fixed moneyness at each date by fitting a simple second order polynomial function,

$$
\ln (P / T)=a(\ln T)^{2}+b(\ln T)+c
$$

with $P$ being the put option price (mid-quote) as a percentage of the underlying futures price and $T$ being the maturity. As discussed in the simulation section, this second-order polynomial fitting presents convenient estimates for the slope and curvature of the term decay plots. To estimate the smoothed term decay plot, we further require that there are at least five distinct maturities at each date and drop those days with smaller cross sections. Of the whole sample, 284 days satisfies this criterion.

Furthermore, since the second-order polynomial fits all the simulated term decay plots very well, the goodness-of-fit on the data provides another criterion on the quality, and synchronicity in particular, of the real data: we would have more confidence on the quality of the data if the data points mostly lie on a smooth second-order polynomial curve. We find that, on most days, the second-order polynomial fits the data well. The average R-square for all the fittings is 0.98. Nevertheless, visual inspection identifies a few days when some maturities deviate significantly from a smoothed term decay curve. 
The R-squares at these days are low as a result. We suspect that these days are more likely to have data measurement errors. We hence use the R-square as yet another criterion to filter the data: we drop any days when any one of the four polynomial regressions (at different moneyness) has a R-square less than 0.80. Finally, we have 273 days left, with an average $R^{2}$ of 0.99 . After all these filtering, we believe that the impact of microstructure effects on our analysis is minimal. Armed with these smoothed term decay plots, we analyze the underlying process followed by the S\&P 500 index.

\section{B. Is There a Jump Component?}

The key indicator of a jump component lies in the slope of the term decay plot for OTM options as the option maturity falls within a month or so. A jump component exists if the term decay plot flattens out (slope approaching zero) as maturity nears.

Figure 5 depicts the two typical shapes of the term decay plots for S\&P 500 index options on April 9th, 1999 (the left panel) and on May 3rd, 2000 (the right panel). The plots follow the same convention as in the simulations (Figures 1, 2, and 4). They represent the two extreme cases that are experienced over the whole sample period. The term decay plots on April 9th, 1999 (left panel) match the shapes generated from a purely continuous process: while the ATM term decay plot looks like a straight line, the OTM term decay plots exhibit strong conacvity and positive slopes at short maturities. The short maturity slope estimates (at $\ln T=-4$ ) are, from top to bottom, $-0.377,1.670,2.458$, and 3.725, and the curvature estimates, $-0.027,-0.574,-0.783$, and -1.100 , corresponding to moneyness levels of $k=0,-3.07 \%,-6.14 \%$, and $-9.20 \%$, respectively. The strongly negative curvature estimates and strongly positive short-maturity slope estimates for OTM options are indicative of an asymptotic $O\left(e^{-1 / T}\right)$ decay rate. Hence, the term decay plots on April 9th, 1999 (left panel) reveal little sign of a jump component.

In contrast, the impacts of a jump component are vividly shown in the term decay plots on May 3rd, 2000 (right panel). The OTM term decay plots obviously flatten out at short maturities. The short maturity slope estimates are, from top to bottom, $-0.604,-0.300,-0.116$, and 0.133 , corresponding to moneyness levels of $k=0,-3.07 \%,-6.14 \%$, and $-9.20 \%$, respectively. While the ATM term 

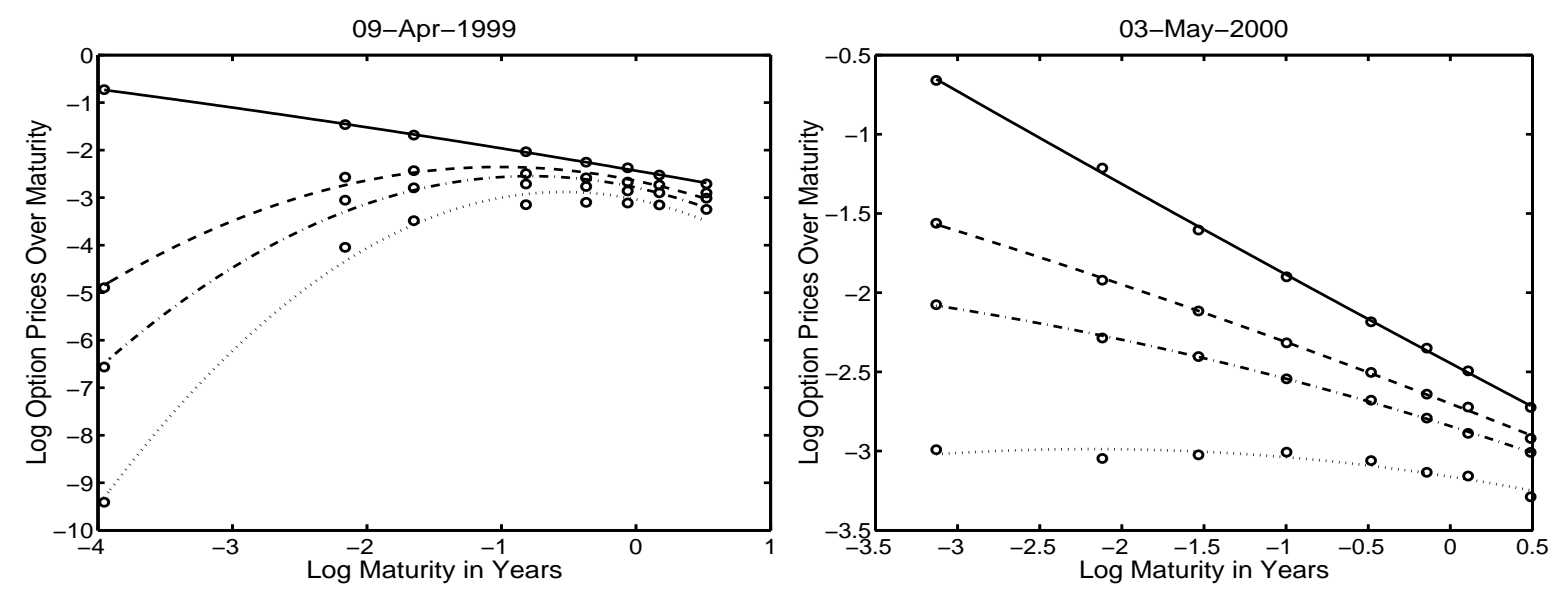

Figure 5. Typical Term Decay Plots for S\&P 500 Index Options

Circles represent data while lines represent quadratic fits. The left panel depicts the term decay plots of S\&P 500 index options on April 9th, 1999. The right panel depicts that on May 3rd, 2000. In each panel, the four lines, from top to bottom, represent moneyness levels at $k=\ln K / F=0$ (solid), $-3.07 \%$ (dashed), $-6.14 \%$ (dash-dotted), and $-9.20 \%$ (dotted).

decay plot is strongly negatively sloped, the OTM plots all have short-maturity slopes close to zero. The curvature estimates for the four term decay plots are all very small, from -0.07 to 0.01 .

On other days, the term decay plots fall between the two extreme cases. Figure 6 illustrates the different shapes of the term decay plots for ATM options (left panel) and for OTM options (right panel, $k=-9.2 \%$ ). Most of the term decay plots for ATM options are strongly negatively sloped, indicating the dominance of an infinite variation component. Furthermore, the majority of the plots for OTM options exhibit some concavity and slightly positive slope at short maturities, indicating the existence of a jump component, possibly interacting with a continuous component. Overall, the existence of a jump component in the movement of S\&P 500 index levels seems to vary significantly over time. Sometimes, its presence is strongly felt in the options market; while at other times its impact is almost non-existent. 

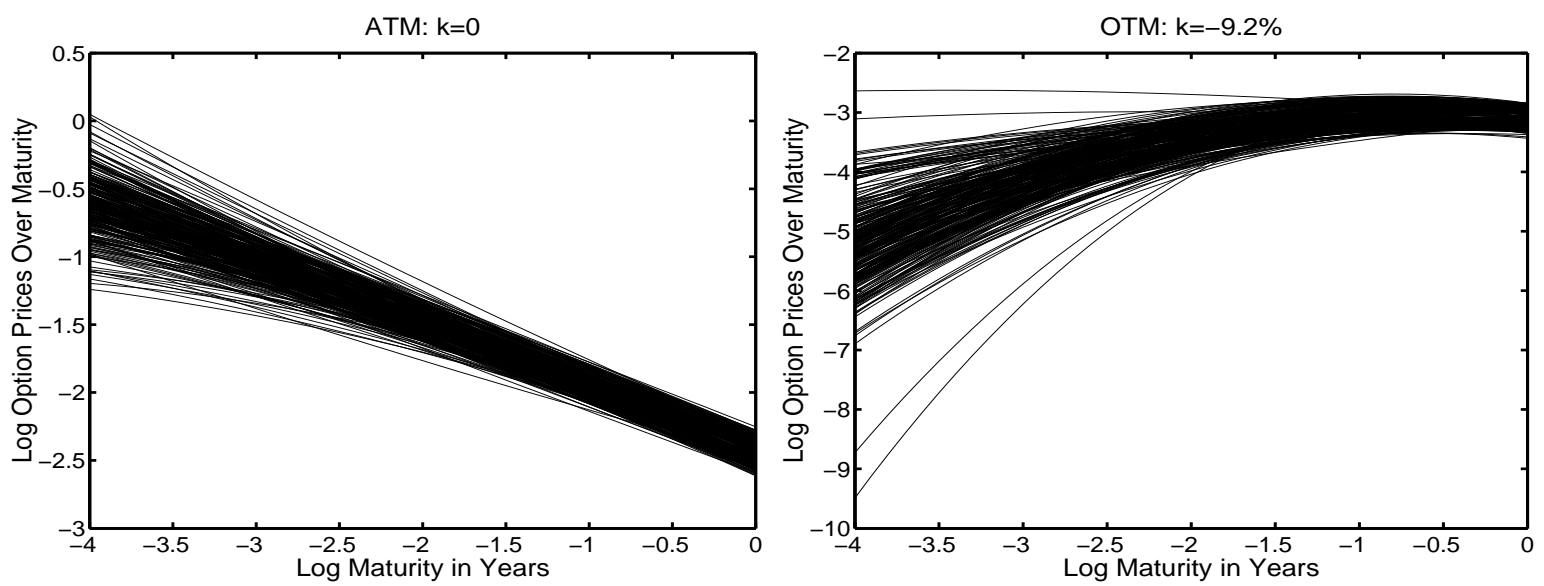

Figure 6. Daily Term Decay Plots for S\&P 500 Index Options

Lines represent smoothed daily term decay plots fox S\&P 500 index options from April 6th, 1999 to May 31st, 2000. The smoothing is performed based on second-order polynomial fits. The left panel depicts the term decay plots for ATM options $(k=0)$. The right panel depicts the term decay plots for OTM options with $k=-9.2 \%$.

\section{Is There a Continuous Component?}

When a continuous martingale component is present, the infinite variation required of it can have a strong impact on the term behavior of ATM option premia. When only a continuous martingale component is present, the term decay plots of ATM options are straight lines with an asymptotic slope of -0.5 . However, since this behavior can also be generated by an infinite variation pure jump component, we need to interpret the term decay plots for ATM options with care. Nevertheless, various pieces of evidence suggest the existence of a continuous martingale component in the S\&P 500 index movement.

The first piece of evidence comes from the term decay plots for ATM options in the left panel of Figure 6. Most of the plots are strongly negatively sloped and look like straight lines. This is strong evidence on the presence of an infinite variation component, which can either a continuous martingale or an infinite variation pure jump process.

The second piece of evidence comes from days such as April 9th, 1999 (left panel, Figure 5), when the presence of a jump component can hardly be detected. For such days, while we cannot identify the presence of a jump component from the OTM options, the presence of an infinite variation component 
is obvious in the ATM options premia. If there does not exist a jump component, the infinite variation component must be a purely continuous process.

Combining the two pieces of evidence together, we conclude that while the presence of a jump component is varying strongly over time, the continuous component has a more steady presence in the options data.

\section{Time Varying Term Decay and Stochastic Volatility}

Figures fig:dailytail and 6 indicate that the daily term decay plots can take on very different shapes. It is important to understand whether such daily variations come from data noise (e.g. microstructure effects) or underly some fundamental movement in the underlying index process. We investigate this issue by analyzing the time series properties of the term decay plots.

Figure 7 draws the time series plots for the slope (left panel) and curvature (right panel) estimates on the term decay plots for both ATM options and OTM options $(\ln (K / F)=-9.2 \%)$. We observe that for ATM options (solid lines), the short-maturity slope estimates (left panel) fluctuate around -0.5 while the curvature estimates (right panel) fluctuate around zero. Hence, the presence of an infinite variation component is strongly and constantly felt. On the other hand, the slope and curvature estimates for the OTM term decay plots (dashed lines) are much more volatile. In particular, during the first couple of days (April 8th and 9th, 1999) of the sample, the OTM term decay plots exhibit strongly positive slopes and strong negative curvatures, indicating that option prices are approaching to zero at a rate much faster than $O(T)$. The decay rate is more in line with $O\left(e^{-1 / T}\right)$, which is the rate implied by a pure continuous process. Indeed, the slope and curvature estimates are so large in magnitude and so far away from the majority estimates of the whole sample that they look like outliers. But further inspection of the data indicates that they are not outliers, but robust data points. In particular, we also find that, during the first two days of our sample, the implied volatility curves at short maturities are essentially flat. Only at relatively long maturities does a skewed pattern in volatility shows up. This is consistent with the implication of pure continuous models with stochastic volatilities, e.g. Heston (93), 

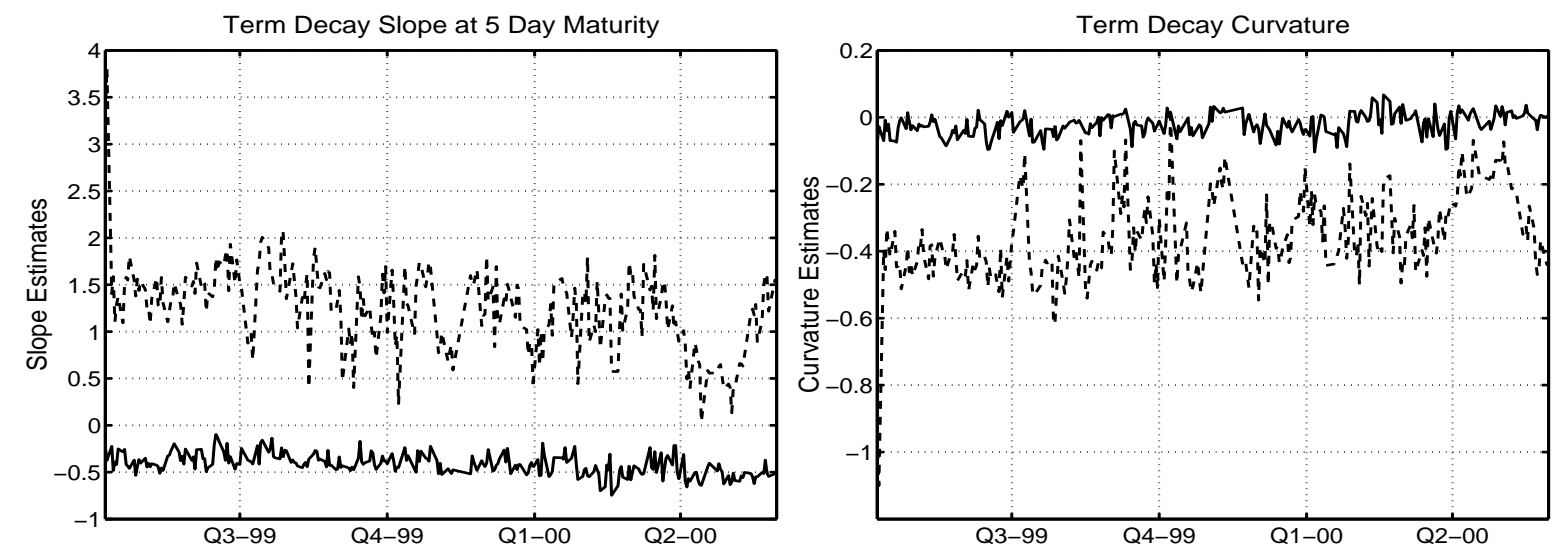

Figure 7. The Slope an Curvature of the Term Decay Plots for S\&P 500 Index Options

Lines in the left panel are the time series slope estimates of the term decay plots at five business day maturity while lines in the right panel are the time series of curvature estimates of the term decay plots. The estimates are based on a quadratic polynomial fit of the data at each day and moneyness. The solid lines represent the estimates on ATM term decay plots $(k=0)$ while the dashed lines are estimates for OTM plots $(k=-9.20 \%)$.

but not with models with a significant presence of a jump component. Our term decay plot reaches the same conclusion.

Nevertheless, the presence of a jump component is evident in days that follows, as the slopes estimates become much smaller, although still positive. The curvature estimates also become much less negative. When we move closer to April 2000, both the slope and the curvature estimates of the OTM term decay plots move very close to zero, implying that the jump component begins to dominate. Furthermore, the temporal patterns of the slope and curvature estimates indicate that the variations are not totally due to purely random noise such as measurement errors, but are indicators of systematic variation in the underlying, such as the presence of stochastic volatility.

Table III reports the summary statistics of these slope and curvature estimates. For ATM options, the sample mean of the slope estimates is -0.413 , slightly lower than implied by a continuous component. The sample mean of the curvature is -0.021 , very close to zero and hence confirming the observations of negatively sloped near-straight lines. For OTM options at $\ln (K / F)=-9.2 \%$, the mean slope estimate is 1.266 , and the curvature estimate is -0.366 , showing the combined effect of 
both a jump component and a continuous component. Furthermore, consistent with the observation from the time series plots, the slope and curvature estimates for the OTM plots are much more volatile than that for the ATM plots. Nevertheless, these fluctuations are not purely random noise, but show significant persistence, as indicated by the moderate first order autocorrelations (from 0.422 to 0.589 ) on the slope and curvature estimates.

The systematic variation of the slope and curvature estimates for the term decay plots points to the presence of stochastic volatility. Depending upon the exact specification, stochastic volatility can generate variations in the term decay plots in different ways. First, given a stationary volatility process, a shock to the volatility level has a larger impact on the short term options than on the long term options. Thus, with an increase in volatility, short-term option prices increase more than long-term option prices do. What this does to the term decay plot is three folds: (1) the overall term decay plot shifts upward; (2) the short-maturity slope of the plot declines, i.e., it becomes less positive or more negative; and (3) the concavity of the plot declines as the curvature estimate becomes less negative or more positive. Thus, holding everything else constant, these three effects will generate a negative correlation between the volatility level and the slope estimate and a positive correlation between volatility and the curvature of the term decay plot.

Nevertheless, stochastic volatility specifications can not only vary the overall volatility level of an asset return, but can also vary the relative composition of its components, say, a jump component and a purely continuous component. The variation of the relative composition of these two components will have yet another impact on the term decay plot. For example, when the volatility level increases, if the relative component of the jump component declines, then two opposite effects will be imposed on the slope of the term decay plot for OTM options. On the one hand, the volatility level increase raises the short end of the term decay plot and makes the slope of the plot less positive. On the other hand, the declining of the jump component makes the diffusion component become the dominating component and hence the slope of the OTM plot more positive. Obviously, such an effect will reduce or even nullify the negative correlation between the volatility level and the slope of the OTM term decay plots. This conflicting impacts, however, will not be observed on the ATM term decay plots because both 
increasing volatility level and increasing the relative composition of the diffusion component make the ATM term decay plot more negatively sloped. The correlation between the volatility level and the ATM term decay slopes may become even more negative.

The jump-diffusion and stochastic volatility model applied in Bakshi, Cao, and Chen (1997) and Bates (1996) falls within this category. The model incorporates three components into the underlying price process: jumps (MJ), diffusion, and stochastic volatility. In both papers, stochastic volatility is generated through the instantaneous variance of the diffusion component, $v_{t}$, which is allowed to follow the square root process of Heston (1993). The arrival rate $(\lambda)$ of the Poisson jump component, however, is assumed to be constant over time. Therefore, under such specifications, as $v_{t}$ increases, the relative composition of the jump component declines.

In the last row of Table III, we report the correlations of the slope and curvature estimates of the term decay plots with the volatility level. The volatility level is proxyed by the ATM implied volatility at the short maturity: $\ln T=-4$. ATM implied volatility at each maturity is obtained via spline interpolation across moneyness. We then fit a second-order polynomial to the ATM implied volatilities (IV) across $\log$ maturities $(\ln T)$,

$$
I V=a(\ln T)^{2}+b(\ln T)+c .
$$

The fitting is reasonably well with an average R-square of 0.92 . The correlations of the volatility level with the term decay slopes are strongly negative, while that with the curvature are strongly positive. Furthermore, the negative correlation between volatility and slopes are stronger for ATM options than for OTM options. Such evidence is qualitatively compatible with the specification in Bakshi, Cao, and Chen (1997) and Bates (1996).

More recently, Bates (2000) and Pan (2002), among others, allow the arrival rate of the Poisson process to be an affine function of the instantaneous variance of the diffusion component: $\lambda_{t}=a+$ $b v_{t}, a, b \in \mathbb{R}^{+}$and hence the arrival rate of the Poisson jump is also allowed to be stochastically time varying. Depending on the magnitude of loading $b$ on the volatility factor, this model can generate either positive or negative correlations between the volatility level and the relative composition of the 
jump component. Thus, the model is even more flexible in generating the correlations observed in the data.

Nevertheless, under all these specifications, the continuous component can totally disappear as $v_{t} \rightarrow 0$, while the jump component has a constant presence. In the former case, the jump intensity $\lambda$ is constant over time; in the latter case, the intensity has a constant component, $a$. The evidence on the term decay plots, however, seems to argue the other way around: while we do observe the jump component disappearing on some days, the presence of a continuous component is constantly felt. Thus, for model design, it seems that a simple role reversal would better capture the evidence from our term decay plots. That is, one can let the arrival rate of the Poisson jump component, $\lambda_{t}$, follow a mean reverting square root process, while the instantaneous variance of the diffusion component can be specified as an affine function of the Poisson intensity: $v_{t}=a+b \lambda_{t}, a, b \in \mathbb{R}^{+}$. This role reversal implies that while the arrival rate of the Poisson process can disappear, the diffusion component always has a constant presence.

Of course, there is no reason that the arrival rate of the Poisson process and the instantaneous variance of the diffusion component should be driven by the same stochastic process. They may very well be driven by separate stochastic forces. Such a specification would also accommodate our evidence and could potentially generate better performance for option pricing. For future research, it is intriguing to investigate the option pricing performance of these alternative specifications against traditional ones.

\section{Concluding Remarks}

We provide a simple test for the nature of the price process of an asset underlying an option. In particular, we map the short maturity behavior of option prices to the type of process the underlying asset price follows. Our analysis of S\&P 500 index options indicates that there are both continuous and jump components in the underlying index process. In particular, we find that while the presence of the jump component varies strongly over time, the presence of the continuous component is constantly 
felt. These observations have interesting implications for specifications of the underlying price process, which can be further explored in future research. 


\section{References}

Aït-Sahalia, Yacine, 2002, Telling from discrete data whether the underlying continuous-time model is a diffusion, Journal of Finance October, forthcoming.

Ait-Sahalia, Yacine, Yubo Wang, and Francis Yared, 2001, Do options markets correctly price the probabilities of movement of the underlying asset?, Journal of Econometrics 102, 67-110.

Andersen, Leif, and Jesper Andreasen, 1999, Jumping smiles, Risk November, 65-68.

Andersen, Torben G., Luca Benzoni, and Jesper Lund, 2002, An empirical investigation of continuoustime equity return models, Journal of Finance 57, 1239-1284.

Bakshi, Gurdip, Charles Cao, and Zhiwu Chen, 1997, Empirical performance of alternative option pricing models, Journal of Finance 52, 2003-2049.

Bates, David, 1991, The crash of 87: Was it expected? The evidence from option markets, Journal of Finance 46, 1009-1044.

Bates, David, 1996, Jumps and stochastic volatility: Exchange rate processes implicit in Deutsche Mark options, Review of Financial Studies 9, 69-107.

Bates, David, 2000, Post-' 87 crash fears in the S\&P 500 futures option market, Journal of Econometrics 94, 181-238.

Bertoin, Jean, 1996, Lévy Processes. (Cambridge University Press Cambridge).

Black, Fisher, and Myron Scholes, 1973, The pricing of options and corporate liabilities, Journal of Political Economy 81, 637-654.

Brenner, Michael, and Marti Subrahmanyam, 1988, A simple formula to compute the implied standard deviation, Financial Analysts Journal 44, 80-83.

Carr, Peter, and Dilip Madan, 1999, Option valuation using the fast fourier transform, Journal of Computational Finance 2, 61-73.

Carr, Peter, and Liuren Wu, 2002, Finite moment logstable process and option pricing, Journal of Finance forthcoming. 
Delbaen, F., and W. Schachermayer, 1994, A general version of the fundamental theorem of asset pricing, Mathematische Annalen 300, 563-520.

Ding, Zhuanxin, and Clive W. J. Granger, 1996, Modeling volatility persistence of speculative returns: A new approach, Journal of Econometrics 73, 185-215.

Duffie, Darrell, Jun Pan, and Kenneth Singleton, 2000, Transform analysis and asset pricing for affine jump diffusions, Econometrica 68, 1343-1376.

Heston, Stephen, 1993, Closed-form solution for options with stochastic volatility, with application to bond and currency options, Review of Financial Studies 6, 327-343.

Jacod, Jean, and Albert N. Shiryaev, 1987, Limit Theorems for Stochastic Processes. (Springer-Verlag Berlin).

Merton, Robert C., 1976, Option pricing when underlying stock returns are discontinuous, Journal of Financial Economics 3, 125-144.

Pan, Jun, 2002, The jump-risk premia implicit in options: Evidence from an integrated time-series study, Journal of Financial Economics 63, 3-50.

Prokhorov, Yurii Vasilevich, and Albert Nikolaevich Shiryaev, 1998, Probability Theory III : Stochastic Calculus. (Springer-Verlag Berlin).

Protter, Philip, 1990, Stochastic Integration and Differential Equations: A New Approach. (Springer Berlin).

Varadhan, S.R.S., 1967, On the behavior of the foundamental solution of the heat equation with variable coefficients, Communications in Pure and Applied Mathematics 20, 431-455. 


\section{Table II}

\section{Slope and Curvature of Simulated Term Decay Plots}

Entries report the slope and curvature estimates (standard errors in parentheses) from simulated term decay plots based on a second order polynomial fit:

$$
\ln (P / T)=a(\ln T)^{2}+b(\ln T)+c,
$$

where $P$ and $T$ denote, respectively, the put option price and the maturity of the option. The polynomial fitting is performed on simulated prices at the $\log$ maturity range of $\ln T=[-4: 0]$, with an equal interval of 0.2. Based on the estimates for the polynomial coefficients $[a, b]$, the slope of the curve is $2 a \ln T+b$ and the curvature, $2 a$. We evaluate the slope at the short end of the maturity: $\ln T=-4$. Also reported is the R-square $\left(R^{2}\right)$ of each polynomial fitting.

\begin{tabular}{|c|c|c|c|c|c|c|}
\hline \multirow[b]{2}{*}{ Model } & \multicolumn{3}{|c|}{$\ln K / F=0$} & \multicolumn{3}{|c|}{$\ln K / F=-9.2 \%$} \\
\hline & slope & Curvature & $R^{2}$ & slope & Curvature & $R^{2}$ \\
\hline & \multicolumn{6}{|c|}{ A. Pure Continuous Models } \\
\hline BS & $\begin{array}{c}-0.497 \\
(0.000)\end{array}$ & $\begin{array}{c}-0.003 \\
(0.000)\end{array}$ & $\begin{array}{c}1.000 \\
-\end{array}$ & $\begin{array}{c}1.988 \\
(0.092)\end{array}$ & $\begin{array}{c}-0.713 \\
(0.044)\end{array}$ & $\begin{array}{c}0.976 \\
-\end{array}$ \\
\hline \multirow[t]{3}{*}{ Heston } & -0.493 & -0.018 & 1.000 & 3.158 & -1.092 & 0.979 \\
\hline & $(0.001)$ & $(0.000)$ & - & $(0.142)$ & $(0.069)$ & - \\
\hline & \multicolumn{6}{|c|}{ B. Pure Jump Models } \\
\hline \multirow[t]{2}{*}{ MJ } & 0.086 & -0.155 & 0.997 & 0.027 & -0.096 & 0.999 \\
\hline & $(0.012)$ & $(0.006)$ & - & $(0.004)$ & $(0.002)$ & - \\
\hline \multirow[t]{2}{*}{ LS } & -0.357 & -0.017 & 1.000 & 0.085 & -0.068 & 0.973 \\
\hline & $(0.001)$ & $(0.000)$ & - & $(0.009)$ & $(0.004)$ & - \\
\hline $\operatorname{LS}(\alpha)$ & \multicolumn{6}{|c|}{ C. Impact of Tail Index under LS Model } \\
\hline \multirow[t]{2}{*}{1.2} & -0.241 & -0.034 & 1.000 & 0.044 & -0.068 & 0.997 \\
\hline & $(0.001)$ & $(0.001)$ & - & $(0.004)$ & $(0.002)$ & - \\
\hline \multirow[t]{2}{*}{1.5} & -0.357 & -0.017 & 1.000 & 0.085 & -0.068 & 0.973 \\
\hline & $(0.001)$ & $(0.000)$ & - & $(0.009)$ & $(0.004)$ & - \\
\hline \multirow[t]{2}{*}{1.9} & -0.474 & -0.005 & 1.000 & 0.605 & -0.170 & 0.973 \\
\hline & $(0.001)$ & $(0.000)$ & - & $(0.040)$ & ( 0.019$)$ & - \\
\hline \multirow[t]{3}{*}{2.0} & -0.498 & -0.003 & 1.000 & 3.255 & -1.098 & 0.980 \\
\hline & $(0.000)$ & $(0.000)$ & - & $(0.149)$ & $(0.072)$ & - \\
\hline & \multicolumn{6}{|c|}{ D. Mixture Models } \\
\hline \multirow[t]{2}{*}{ MJD } & -0.347 & -0.019 & 1.000 & 0.042 & -0.065 & 0.993 \\
\hline & $(0.005)$ & $(0.002)$ & - & $(0.007)$ & $(0.003)$ & - \\
\hline \multirow[t]{2}{*}{ LSD } & -0.452 & -0.005 & 1.000 & 0.369 & -0.163 & 0.972 \\
\hline & $(0.001)$ & $(0.000)$ & - & $(0.014)$ & $(0.007)$ & - \\
\hline
\end{tabular}




\section{Table III}

\section{Slope and Curvature of the Term Decay Plots on S\&P 500 Index Options}

Entries report the summary statistics on the slope and curvature estimates from the smoothed term decay plots on S\&P 500 index options. The smoothing is based on a second order polynomial fit,

$$
\ln (P / T)=a(\ln T)^{2}+b(\ln T)+c,
$$

where $P$ and $T$ denote, respectively, the put option price (as percentages of the underlying futures price level) and the maturity of the option. Based on the estimates for the polynomial coefficients, the slope of the term decay plot is $2 a \ln T+b$ and the curvature, $2 a$. We evaluate the slope at the short end of the maturity: $\ln T=-4$. The data are daily from April 6th, 1999 to May 31st, 2000, 273 business days. "Mean, Std, Auto, Skewness, Kurtosis" denote, respectively, the sample average, standard deviation, first order autocorrelation, skewness, and excess kurtosis of the estimates. The last row, $\operatorname{Corr}(\cdot, I V)$, measures the correlations of these slope and curvature estimates with the volatility level, which is proxyed by the smoothed ATM implied volatility at short maturity: $\ln T=-4$.

\begin{tabular}{lccccc}
\hline \hline \multirow{2}{*}{ Stats } & \multicolumn{2}{c}{$\ln K / F=0$} & & \multicolumn{2}{c}{$\ln K / F=-9.2 \%$} \\
\cline { 2 - 3 } \cline { 5 - 6 } & Slope & Curvature & & Slope & Curvature \\
Mean & -0.413 & -0.021 & & 1.266 & -0.366 \\
Std & 0.114 & 0.032 & & 0.441 & 0.128 \\
Auto & 0.506 & 0.422 & & 0.586 & 0.559 \\
Skewness & 0.131 & -0.199 & & 0.569 & -0.484 \\
Kurtosis & -0.102 & -0.293 & & 4.846 & 4.598 \\
Corr $(\cdot, I V)$ & -0.749 & 0.540 & & -0.584 & 0.515 \\
& & & & \\
\hline \hline
\end{tabular}

